\title{
МАГНЕТИТ С МОДИФИЦИРОВАННОЙ ПОВЕРХНОСТЬЮ ДЛЯ ВОДООЧИСТКИ
}

\author{
Чан Туан Хоанг', \\ cungbinh9327@gmail.com
Юрмазова Татьяна Александровна', yur-tatyana@yandex.ru \\ Вайтулевич Елена Анатольевна', \\ rhodamine@tpu.ru \\ 1 Национальный исследовательский Томский политехнический университет, \\ Россия, 634050, г. Томск, пр. Ленина, 30.
}

\begin{abstract}
Актуальность. В последнее время возрос интерес к синтезу и модификации магнетита, что связано с возможностью применения его в качестве сорбента для защиты окружающей среды, например, для очистки сточных вод от органических соединений, лекарственных препаратов и от неорганических ионов тяжелых металлов. Необходимость повысить эффективность сорбционных процессов и изучить механизм процессов сорбции магнетита делает актуальной эту проблему.

Цель: определение сорбционных возможностей нанопорошков магнетита, полученного химическим способом с модифицированной поверхностью по отношению к неорганическим и органическим ионам, а также лекарственным препаратам для дальнейшего использования в процессах водоочистки.

Объект: магнитные нанопорошки на основе магнетита $\mathrm{Fe}_{3} \mathrm{O}_{4}$ С модифицированной поверхностью цитрат-ионами и диоксидом кремния.

Методы: рентгенофазовый анализ, атомно-эмиссионная спектрометрия с индуктивно-связанной плазмой, метод тепловой десорбции азота, индикаторный метод.

Результаты. Магнитные нанопорошки на основе магнетита $\mathrm{Fe}_{3} \mathrm{O}_{4}$, покрытые цитрат-ионами (HЧМ) и диоксидом кремния (HчM/SiO $)_{2}$, были получены для сорбции органических и неорганических соединений из водных растворов. Изучены их состав и магнитные свойства. Показано, что полученный состав для магнетита с покрытием диоксида кремния соответствует кристаллической и аморфной фазе $\mathrm{Fe}_{3} \mathrm{O}_{4}$ и $\mathrm{SiO}_{2}$, соответственно. Кроме того, определена намагниченность насыщения $\mathrm{M}_{5}$ и средний поверхностный диаметр частиц. Изотермы сорбции органических и неорганических соединений на поверхности полученных нанопорошков описываются уравнением Ленгмюра, что свидетельствует об образовании мономолекулярного слоя на поверхности сорбентов. Определена максимальная сорбционная емкость порошков магнетита, модифицированного диоксидом кремния, она составляет для $\mathrm{Ni}^{2+}-22,3$ мг/г, метиленового голубого 14,7 мг/г, доксорубицина - 18,8 мг/г. С помощью индикаторного метода определен заряд активных центров поверхности магнетита. Полученные результаты дают возможность моделировать процессы сорбции органических, неорганических веществ и лекарственных препаратов в процессах водоочистки и утилизации химических соединений на фармацевтических предприятиях и в онкологических центрах.
\end{abstract}

\section{Ключевые слова:}

Адсорбция, магнитные наночастицы, модификация поверхности, органические и неорганические ионы, заряд активных центров поверхности.

\section{Введение}

В настоящее время развитие промышленности приводит к усилению антропогенного воздействия на окружающую среду. В водные бассейны попадают гальваностоки, содержащие различные ионы, такие как: $\mathrm{Pb}^{2+}, \mathrm{Ni}^{2+}, \mathrm{CrO}_{4}{ }^{2-}$, и др., которые имеют высокую токсичность [1-3]. Сточные воды предприятий текстильной промышленности содержат большие концентрации красителей, превышающие ПДК $[4,5]$. Кроме того, для лечения различных заболеваний применяются высокотоксичные препараты, неиспользованные остатки которых необходимо утилизировать перед сбрасыванием в сточные воды, потому что попадание этих препаратов пагубно влияет на водную экосистему [6, 7]. $\mathrm{C}$ точки зрения риска для окружающей среды требуется целевая система водоочистки от вышеприведенных соединений. В процессах водоочистки используют различные методы [8-11], в том числе сорбционный. Создание универсального сорбента для очистки сточных вод от химических соедине- ний различного состава и свойств является актуальным направлением. В последнее время вырос интерес к магнетиту для применения его в процессах водоочистки и биомедицине. Если работ, связанных с применением магнетита в биомедицине, много [12-14], то работы, связанные с использованием его в процессах водоочистки, практически отсутствуют [15-17]. Магнетит является перспективным сорбентом из-за высокой сорбционной способности, невысокой чувствительности к окислению и большой стабильности [18]. Кроме того, нанопорошки магнетита легко отделяются после окончания сорбции внешним магнитным полем, что важно для восстановления или повторного использования нанопорошков магнетита [16]. Получение магнетита химическим способом исключает влияние примесей, которые присутствуют в природном минерале. Это необходимо для изучения механизма процессов сорбции, который до конца не изучен. Часто для увеличения сорбционной емкости, гидрофильности и стабильности, магнетит 
модифицируют тетроэтоксисиланом, хитазаном, полиэтиленгликолем и др. [19-22]. Использование для модифицирования наночастиц магнетита тетраэтоксисилана (ТЭОС) не только помогает улучшить эти свойства, но также облегчает модификацию поверхности из-за доступности многочисленных силанольных групп (- $\mathrm{SiOH})$ на поверхности.

Несмотря на многочисленные приведенные публикации, посвященные синтезу, модифицированию и изучению различных свойств нанопорошков магнетита, в них отсутствует комплексный подход к изучению взаимосвязи процессов формирования порошков, модифицирования их поверхности и сорбционных свойств и механизма сорбции.

Данная статья посвящена изучению взаимосвязи между синтезом нанопорошков магнетита, полученных химическим способом, условиями модифицирования их поверхности и исследованию физико-химических свойств; изучению сорбционной возможности по отношению к органическим, неорганическим ионам и лекарственным препаратам для дальнейшего использования его в процессах водоочистки.

\section{Экспериментальная часть}

Реактивы

Для синтеза магнетита в работе использовали следующие реактивы: $\mathrm{FeCl}_{3} \cdot 6 \mathrm{H}_{2} \mathrm{O}, \mathrm{FeCl}_{2} \cdot 4 \mathrm{H}_{2} \mathrm{O}$, $\mathrm{NaOH}$ (Реахим, Россия). Для стабилизации и модифицирования поверхности частиц использовали лимонную кислоту $\mathrm{C}_{6} \mathrm{H}_{8} \mathrm{O}_{7}$ и тетраэтоксисилан (ТЭОС) (Химпром, Россия). В качестве адсорбатов были выбраны органические соединения: катионный краситель метиленовый голубой (MГ) $\mathrm{C}_{16} \mathrm{H}_{18} \mathrm{ClN}_{3} \mathrm{~S} \cdot \mathrm{HCl}$ (х.ч., PЕAХИМ, Россия), анионный краситель эозин $\mathrm{Na}_{2}\left[\mathrm{C}_{20} \mathrm{H}_{6} \mathrm{O}_{5} \mathrm{Br}_{4}\right]$ (х.ч., РЕАХИМ, Россия) и противоопухолевый препарат доксорубицин (DOX) $\mathrm{C}_{27} \mathrm{H}_{29} \mathrm{NO}_{11} \cdot \mathrm{HCl}$ (Верофарм, Россия); и неорганические ионы $\mathrm{CrO}_{4}{ }^{2-}, \mathrm{Ni}^{2+}$, полученные при растворении солей $\mathrm{NiSO}_{4}, \mathrm{~K}_{2} \mathrm{CrO}_{4}$ (х.ч., РЕАХИМ, Россия). Все реактивы марки «х.ч.» использовались без дальнейшей очистки.

\section{Условия синтеза нанопорошков магнетита}

Коллоидный раствор наночастиц магнетита получали путем химического соосаждения хлоридов железа (II) и (III) в соотношении 1 к 2 в щелочной среде [23]. Их синтез осуществляли согласно следующей реакции:

$2 \mathrm{FeCl}_{3}+\mathrm{FeCl}_{2}+8 \mathrm{NaOH} \rightarrow \mathrm{Fe}_{3} \mathrm{O}_{4}$ (тв.) $+4 \mathrm{H}_{2} \mathrm{O}+8 \mathrm{NaCl}$.

Раствор нагревался до температуры $60{ }^{\circ} \mathrm{C}$ при интенсивном перемешивании со скоростью 2000 об/мин. Удаление кислорода достигалось пропусканием азота через раствор на протяжении реакции синтеза. При достижении указанной температуры в раствор вливали нагретый 1,5 M NaОН, что вызывало повышение $\mathrm{pH}$ до 11. Для устранения низкой агрегативной устойчивости и сорбционной способности из-за отсутствия заряда поверхности магнетита [20] через 30 минут непре- рывного нагрева в раствор вводили лимонную кислоту. После завершения синтеза раствор декантировали, промывали порошки дистиллированной водой. Эту процедуру повторяли до нейтрального $\mathrm{pH}$, а затем порошки осаждали внешним магнитным полем и сушили при комнатной температуре на воздухе.

Синтезированные порошки магнетита, покрытого цитрат-ионами (НЧМ), были разделены на две части. Первую использовали в качестве сорбента, вторую часть порошков модифицировали ТЭОС для покрытия поверхности НЧМ диоксидом кремния $\left(\mathrm{HЧM} / \mathrm{SiO}_{2}\right)$ [24], для чего водный раствор, содержащий НЧМ 4г/100мл, перемешивали в течение нескольких минут при ультразвуковом воздействии (400 Вт, 50-60 Гц). Модифирование поверхности наночастиц осуществляли путем введения в полученный коллоидный раствор спиртового раствора ТЭОС (отношение $\mathrm{Fe}_{3} \mathrm{O}_{4}$ и ТЭОС составляло $2: 1)$. Химические превращения осуществлялись согласно следующим реакциям:

$$
\begin{gathered}
\mathrm{Si}\left(\mathrm{OC}_{2} \mathrm{H}_{5}\right)_{4}+4 \mathrm{H}_{2} \mathrm{O} \rightarrow \mathrm{Si}(\mathrm{OH})_{4}+4 \mathrm{C}_{2} \mathrm{H}_{5} \mathrm{OH}, \\
\mathrm{Si}(\mathrm{OH})_{4} \rightarrow \mathrm{SiO}_{2}+2 \mathrm{H}_{2} \mathrm{O} .
\end{gathered}
$$

После этого суспензию выдерживали при перемешивании и нагревании в течение 2 часов при $90{ }^{\circ} \mathrm{C}$. После остывания до комнатной температуры полученный раствор осаждали в магнитном поле, промывали дистиллированной водой до нейтрального $\mathrm{pH}$ и после отделения жидкой фазы сушили на воздухе при комнатной температуре.

\section{Методы исследования}

Состав синтезированных нанопорошков магнетита определяли рентгенографически на дифрактометре SHIMADZU XRD-6000 (Япония) с использованием компьютерной базы данных рентгеновской порошковой дифрактометрии PDF4+ Международного центра дифракционных данных (ICDD, Denver, USA).

Дополнительно проводили анализ формы частиц, структуры агрегатов и распределения наночастиц магнетита по размерам с использованием электронного микроскопа «JEM-2100F» (Япония).

Автоматизированный комплекс Магнитометр Н-04 использовали для исследования магнитных характеристик в импульсных магнитных полях синтезированных нанопорошков.

При исследовании заряда поверхности магнитных порошков был применен индикаторный метод [25] с использованием катионного красителя $\mathrm{C}_{16} \mathrm{H}_{18} \mathrm{ClN}_{3} \mathrm{~S} \cdot \mathrm{HCl}(\mathrm{M \Gamma})$ и анионного красителя эозина $\mathrm{Na}_{2}\left[\mathrm{C}_{20} \mathrm{H}_{6} \mathrm{O}_{5} \mathrm{Br}_{4}\right]$.

Методом тепловой десорбции азота с помощью анализатора Sorbi-3M (Россия) определяли площадь удельной поверхности образцов $S_{\text {уд }}$.

Исследовали сорбционные свойства магнетита. В качестве адсорбатов были выбраны вышеприведенные красители, противоопухолевый препарат DOX и неорганические вещества $\mathrm{NiSO}_{4}, \mathrm{~K}_{2} \mathrm{CrO}_{4}$. Оптическую плотность растворов определяли с ис- 
пользованием спектрофотометра «ApelPD-303UV》 (Япония) в максимумах полос поглощения: метиленовый голубой - 660 нм, эозин -490 нм, доксорубицин - 490 нм. Концентрацию ионов $\mathrm{CrO}_{4}^{2-}$, $\mathrm{Ni}^{2+}$ определяли с 1,5-дифенилкарбазидом и диметилглиоксимом, соответсвенно, по стандартным методикам [26].

Сорбционную емкость $A_{e}\left(\mathrm{мг} \cdot \Gamma^{-1}\right)$ определяли по формуле [27]:

$$
A_{e}=\frac{\left(C_{0}-C_{e}\right) V}{m},
$$

где $A_{e}$ - равновесная сорбционная емкость, мг·Г $\Gamma^{-1}$;

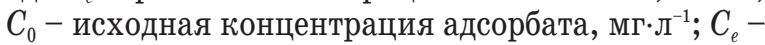
равновесная концентрация адсорбата, мг·л ${ }^{-1} ; V-$ объем адсорбата, л; $m$ - масса адсорбента, г.

\section{Результаты исследования и их обсуждение}

Для определения фазового состава полученных нанопорошков на рис. 1 представлены рентгеновские дифрактограммы образцов НЧМ и $\mathrm{HЧM} / \mathrm{SiO}_{2}$.

Дифракционные рефлексы, наблюдаемые при $2 \theta=29,57^{\circ} ; 34,56^{\circ} ; 41,30^{\circ} ; 47,96^{\circ} ; 49,90^{\circ}$ и $52,15^{\circ}$ для образцов, отнесены к кристаллической фазе магнетита. Эти дифракционные максимумы характерны для плоскостей (220), (311), (400), (511) и (440), соответственно. Таким образом, единственной кристаллической фазой, присутствующей в образцах, является магнетит.

При этом на рис. 1 , б для образцов $\mathrm{HЧM} / \mathrm{SiO}_{2}$ наблюдается широкий рефлекс около $20-28^{\circ}$, который относится к аморфной оболочке из $\mathrm{SiO}_{2}$, остальные дифракционные рефлексы относятся к характерным рефлексам кубической структуры нанопорошков $\mathrm{Fe}_{3} \mathrm{O}_{4}$ и совпадают с таковыми для НЧМ. При этом дифракционные рефлексы до 5 \% , которые могли бы быть связанны с наличием в образцах примесей, отсутствуют. Таким образом, согласно полученным данным, состав для образцов $\mathrm{HЧM} / \mathrm{SiO}_{2}$ соответствует кристаллической $\mathrm{Fe}_{3} \mathrm{O}_{4}$ и аморфной $\mathrm{SiO}_{2}$ фазе.
Средний поверхностный диаметр НЧМ и $\mathrm{HЧM} / \mathrm{SiO}_{2}$, рассчитанный в соответствии с уравнением Дебая-Шеррера, составил 16 и 20 нм, соответственно.

На рис. 2 показаны фотографии образцов двух серий, полученные с помощью просвечивающей электронной микроскопии. Образцы порошков состоят из частиц с почти сферической формой (рис. 2). Наночастицы НЧМ имеют размер приблизительно 10-20 нм, что указывает на относительную однородность синтезированых порошков, согласно данной методике (рис. $2, a$ ). Полученный результат согласуется с результатом работы [17].

Как видно из рис. 2, б, полученные $\mathrm{HЧM} / \mathrm{SiO}_{2}$ являются одиночными близкими по размерам частицами, состав ядра которых соответствует $\mathrm{Fe}_{3} \mathrm{O}_{4}$. Все частицы покрыты однородной светлой оболочкой толщиной до 2 нм из диоксида кремния согласно микроанализу. Средний поверхностный диаметр $\mathrm{HЧM} / \mathrm{SiO}_{2}$ составляет около 20 нм.

Таким образом, синтезированные НЧМ достаточно малы по размеру, это связано с тем, что ионы цитрата из раствора адсорбируются на частицах магнетита, определяя отрицательный заряд на каждой частице, который препятствует укрупнению и агломерации частиц по электростатическому взаимодействию. Таким же образом заряженные ионы щелочного раствора ТЭОС в качестве поверхностно-активного вещества образуют оболочку вокруг каждой частицы магнетита, покрывая цитрат ионы на поверхности НЧМ.

Зависимости гистерезиса НЧМ и $\mathrm{HЧM} / \mathrm{SiO}_{2}$ в порошковом состоянии показаны на рис. 3 .

По данным, представленным на рис. 3 , для нанопорошков магнетита отсутствует петля гистерезиса. Намагниченность насыщения $M_{s}$ составляет $92 \mathrm{~A} \cdot \mathrm{м}^{2} /$ кг для частиц, покрытых цитрат-ионами, и $57 \mathrm{~A} \cdot \mathrm{м}^{2} /$ кг для частиц, покрытых диоксидом кремния. Значение намагниченности насыщения наночастиц становится меньше после модифицикации диоксидом кремния, который экранирует

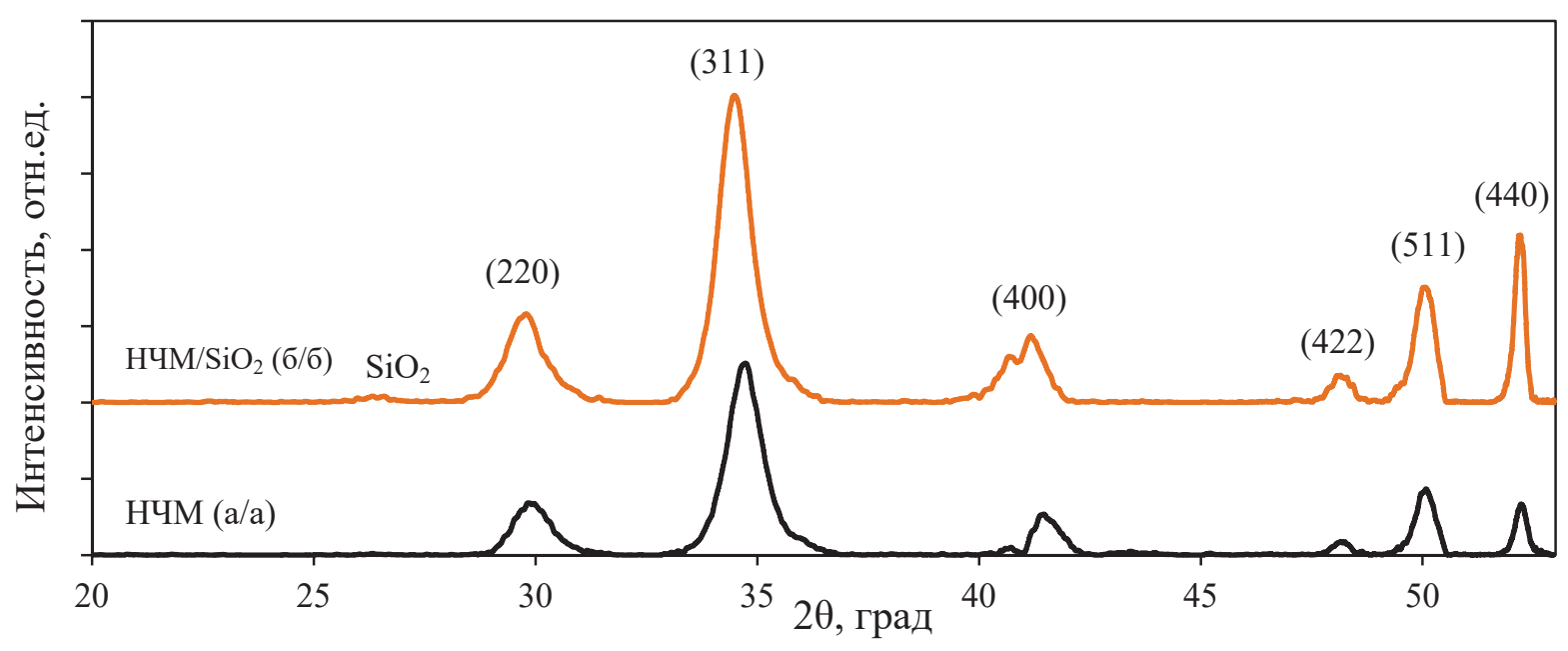

Рис. 1. Рентгеновские дифрактограмлы порошков магнетита, покрытых иитрат-ионами (а) и диоксидом кремния (б)

Fig. 1. XRD patterns of powder coated with citrate ions (a) and silicon dioxide (b) 


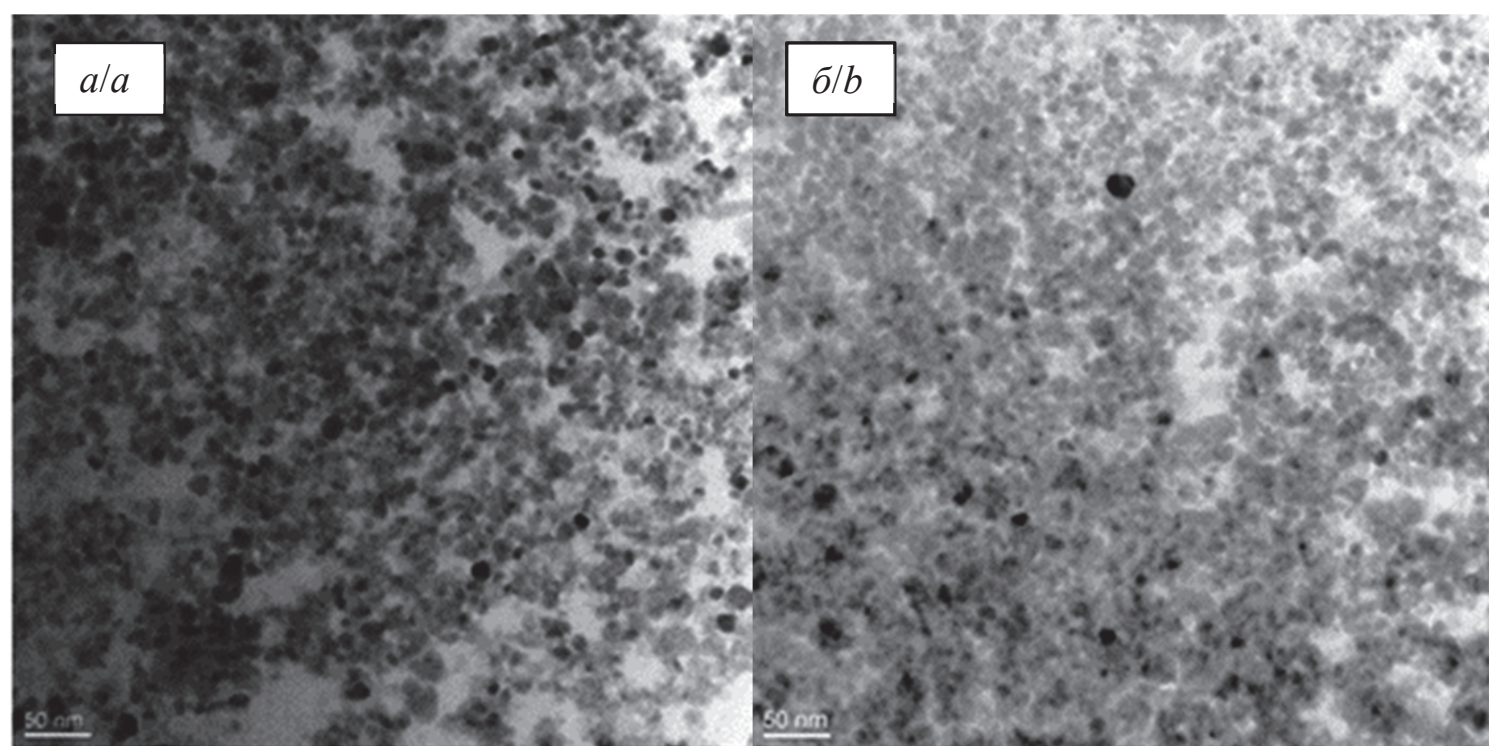

Pис. 2. Фотографии нанопорошков магнетита, покрытых иитрат-ионами (а) и диоксидом кремния (б), полученные с помощью просвечивающей электронной микроскопии

Fig. 2. TEM images of nanopowders coated with citrate ions (a) and silicon dioxide (б)

магнетит от внешнего магнитного поля. Значения намагниченности насыщения $\mathrm{HЧM} / \mathrm{SiO}_{2}$ практически совпадает со значениями в работах других авторов $[1,14,17]$.

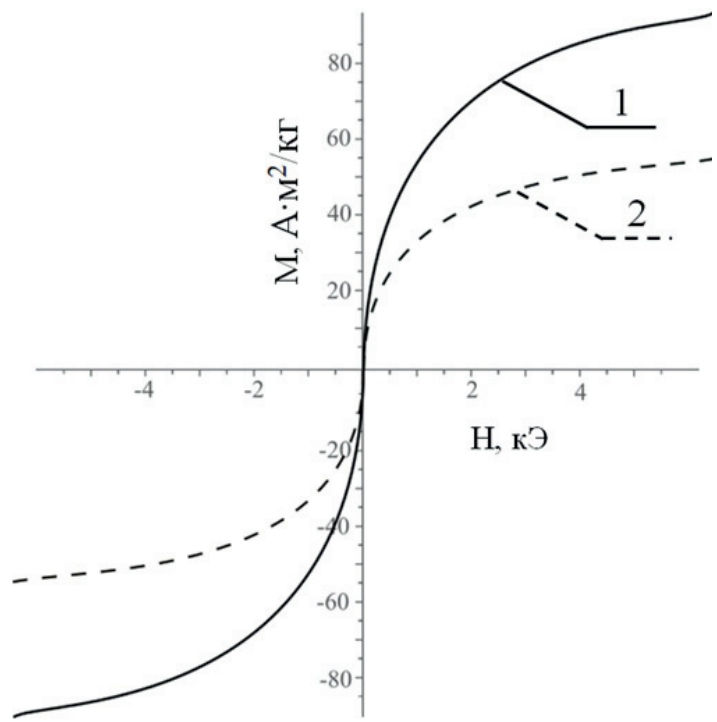

Pис. 3. Зависимость намагниченности от напряженности магнитного поля при комнатной температуре для нанопорошков магнетита, покрытых иитрат-ионами (1) и диоксидом кремния (2)

Fig. 3. Dependence of magnetization of nanopowders MNP (1), $\mathrm{MNP} / \mathrm{SiO}_{2}(2)$ on applied magnetic field at room temperature

Площадь удельной поверхности НЧМ и $\mathrm{HЧM} / \mathrm{SiO}_{2}$ составила 115,4 и $130,5 \mathrm{~m}^{2} / \Gamma$, соответственно. Увеличение площади удельной поверхности для $\mathrm{HЧM} / \mathrm{SiO}_{2}$ объясняется присутствием диоксида кремния на поверхности частиц. Средний размер пор составил 3,0 и 3,8 нм для НЧМ и $\mathrm{HЧM} / \mathrm{SiO}_{2}$, соответственно. Таким образом, синтезированные нанопорошки с такими параметрами обладают высокой адсорбционной способностью при удалении загрязняющих веществ или при адсорбции лекарственных препаратов.

Одним из важных факторов, определяющих сорбционное равновесие, является температура. Процесс сорбции DOX на $\mathrm{HYM} / \mathrm{SiO}_{2}$ изучали при температурах 293, 308 и 323 К. Результаты представлены на рис. 4 , из которого видно, что с ростом температуры максимальная сорбционная ёмкость уменьшается, это указывает на экзотермический процесс адсорбции DOX.

Скорость адсорбции DOX была проанализирована с помощью уравнений псевдо-первого $\left(\ln \left(A_{e}-A_{t}\right)\right.$ от $\left.t\right)$ и псевдо-второго порядка $\left(t / A_{t}\right.$ от $\left.t\right)$ [27]. Результаты расчёта приведены в табл. 1.

Данные зависимости относятся к уравнениям псевдо-второго порядка на основании более высокого значения коэффициента корреляции 0,999 . Следовательно, скорость адсорбции определяется не только скоростью диффузионных процессов, но и скоростью химического взаимодействия между адсорбатом и адсорбентом.

Таблица 1. Параметры уравнений псевдо-первого и псевдо-второго порядка для адсорбции доксорубицина при раз Hыx температурах

Table 1. Parameters of pseudo-first-order and pseudo-second-order equations for doxorubicin adsorption at different temperatures

\begin{tabular}{|c|c|c|c|c|c|c|c|}
\hline \multirow{2}{*}{ 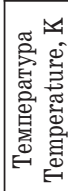 } & \multirow{2}{*}{$\begin{array}{l}A_{e, \text { exp }}, \\
\mathrm{M \Gamma} \cdot \Gamma^{-1} \\
\mathrm{mg} \cdot \mathrm{g}^{-1}\end{array}$} & \multicolumn{3}{|c|}{$\begin{array}{l}\text { Модель пседо-первого } \\
\text { порядка } \\
\text { Pseudo-first-order model }\end{array}$} & \multicolumn{3}{|c|}{$\begin{array}{c}\text { Модель пседо-второго } \\
\text { порядка } \\
\text { Pseudo-second-order model }\end{array}$} \\
\hline & & $\begin{array}{c}k_{1}, \\
\mathrm{\tau}^{-1} \mathrm{~h}^{-1}\end{array}$ & $\begin{array}{l}A_{e, \mathrm{cal}}, \\
\mathrm{M} \Gamma \cdot \Gamma^{-1} \\
\mathrm{mg} \cdot \mathrm{g}^{-1}\end{array}$ & $R^{2}$ & $\begin{array}{c}k_{2}, \\
\Gamma \cdot \mathrm{M}^{-1} \cdot \mathrm{q}^{-1} \\
\mathrm{~g} \cdot \mathrm{mg}^{-1} \cdot \mathrm{h}^{-1}\end{array}$ & $\begin{array}{l}A_{e, \mathrm{cal}}, \\
\mathrm{M} \cdot \mathrm{\Gamma}^{-1} \\
\mathrm{mg} \cdot \mathrm{g}^{-1}\end{array}$ & $R^{2}$ \\
\hline 293 & 14,45 & 0,45 & 7,34 & 0,87 & 0,20 & 14,81 & 0,99 \\
\hline 308 & 13,36 & 0,53 & 7,16 & 0,83 & 0,25 & 13,68 & 0,99 \\
\hline 323 & 12,48 & 0,48 & 4,63 & 0,88 & 0,39 & 12,66 & 0,99 \\
\hline
\end{tabular}




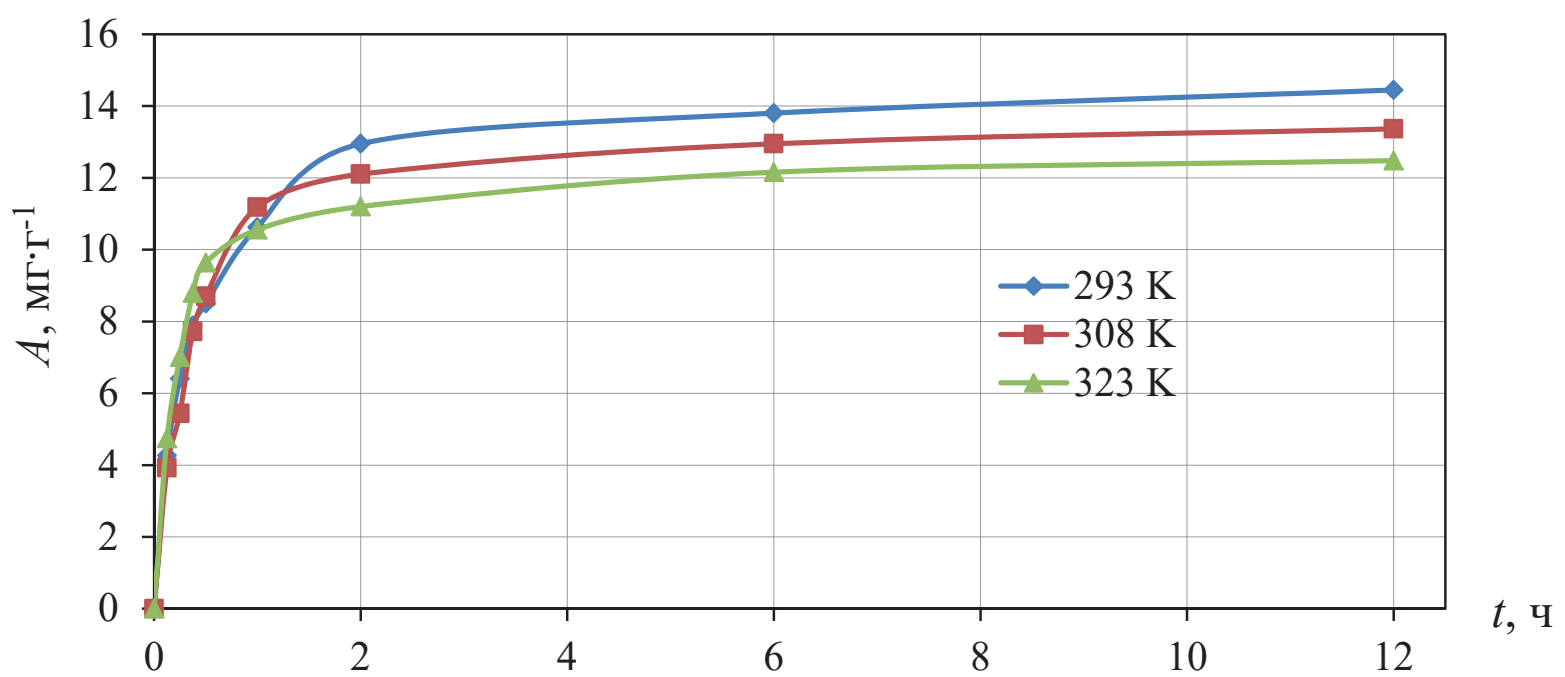

Pис. 4. Зависимость сорбиионной емкости доксорубицина на нанопорошках магнетита, покрытых диоксидом кремния, от времени при разных телпературах

Fig. 4. Dependence of doxorubicin adsorption capacity on magnetite nanopowders coated with silicon dioxide on time at different temperatures

Рассчитанные константы были использованы для определения энергии активации $E_{a}$, которая составила 18,2 кДж/моль. Полученное значение $E_{a}$ является промежуточным между энергиями активации диффузионного (5-10 кДж/моль) и хемосорбционного процессов ( $\geq 40$ кДж/моль) [28].

Оптимальное время достижения сорбционного равновесия при комнатной температуре определили на основании зависимости адсорбционной ёмкости от времени. Оно составило приблизительно 12 ч для всех адсорбатов. При данном времени были проведены все последующие эксперименты по адсорбции.

На рис. 5-7 представлены изотермы адсорбции органических и неорганических ионов.

Как видно из рис. $5-7, \mathrm{M \Gamma}, \mathrm{DOX}, \mathrm{Ni}^{2+}$ сорбируются как на НЧМ так и на $\mathrm{H} Ч М / \mathrm{SiO}_{2}$, а эозин и
$\mathrm{CrO}_{4}{ }^{2-}$ практически не сорбируются. Данный результат свидительствует о том, что активные центры поверхности $\mathrm{HЧM} \mathrm{и} \mathrm{HЧM} / \mathrm{SiO}_{2}$ заряжены отрицательно. В работе [20] был измерен $\zeta$-потенциал немодифицированных наночастиц магнетита, который оказался практически близок к нулю. Таким образом, модифицирование наночастиц магнетита цитрат-ионами и диоксидом кремния изменяет нулевой заряд поверхности магнетита на отрицательный.

Высокая адсорбция МГ обусловлена силами притяжения между катионами красителя, который диссоциирует согласно уравнению:

$$
\mathrm{C}_{16} \mathrm{H}_{18} \mathrm{ClN}_{3} \mathrm{~S} \cdot \mathrm{HCl} \rightarrow\left[\mathrm{C}_{16} \mathrm{H}_{18} \mathrm{ClN}_{3} \mathrm{~S}\right] \mathrm{H}^{+}+\mathrm{Cl}^{-},
$$

и отрицательными зарядами адсорбирующей поверхности частиц. Отрицательный заряд поверхности обуславливает адсорбционную способность и

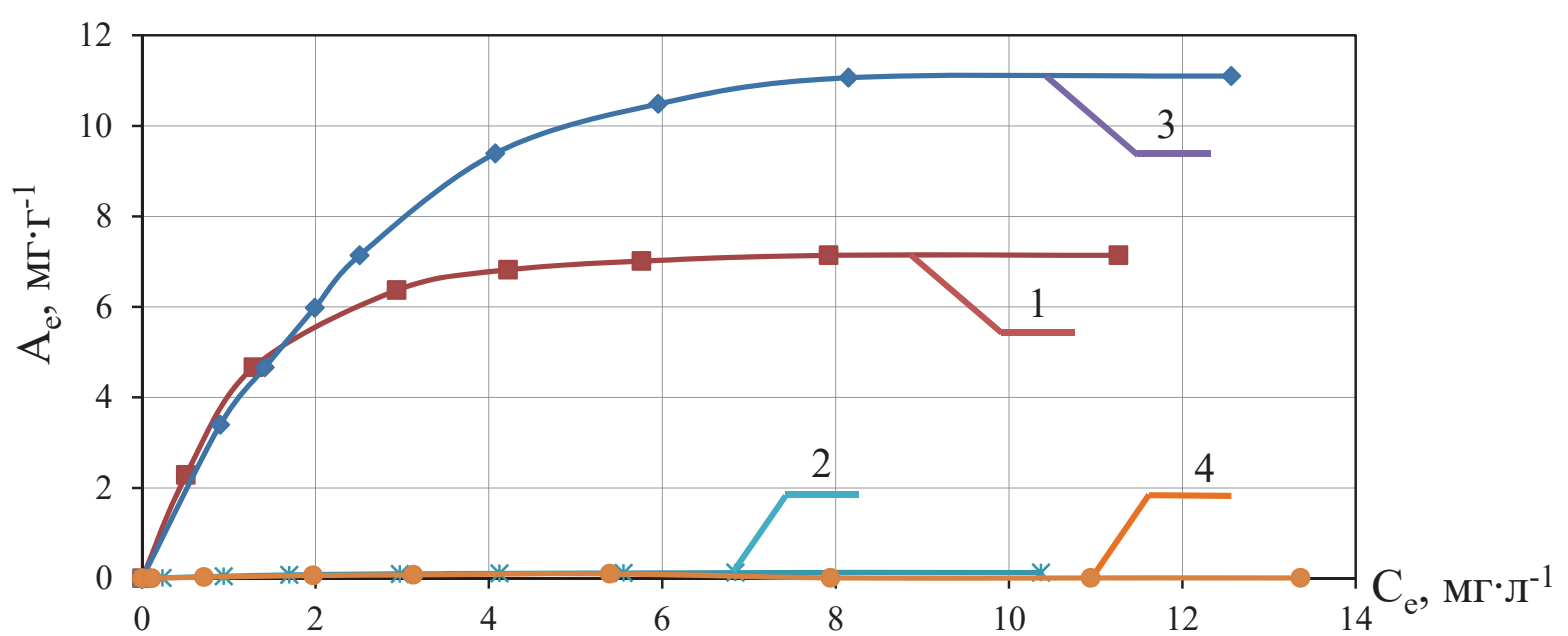

Рис. 5. Изотермы адсорбиии метиленового голубого (1) и эозина (2) на магнетите, покрытом иитрат-ионами; метиленового голубого (3) и эозина (4) на магнетите, покрытом диоксидом кремния

Fig. 5. Adsorption isotherms of methylene blue (1) and eosin (2) on magnetite coated with citrate ions; methylene blue (3) and eosin (4) on magnetite coated silicon dioxide 


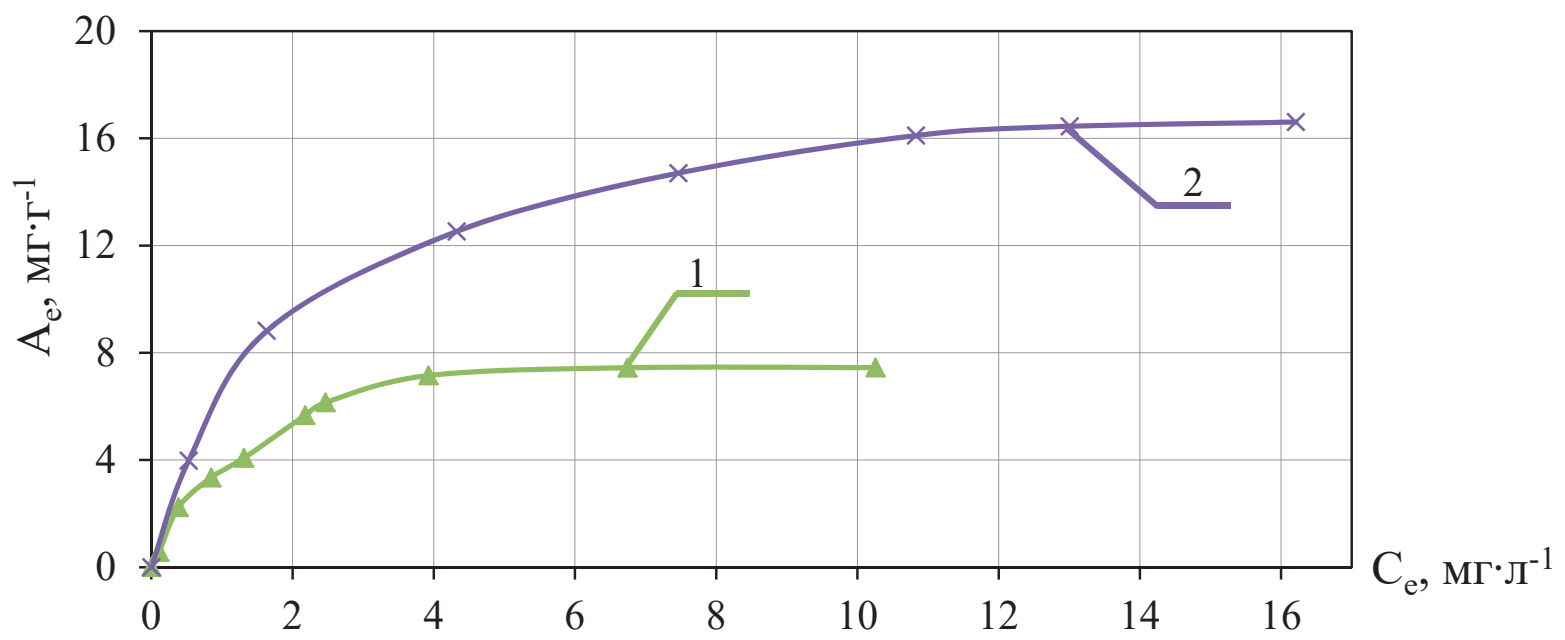

Pис. 6. Изотерлы адсорбции доксорубицина на магнетите, покрытом иитрат-ионами (1) и диоксидом кремния (2)

Fig. 6. Adsorption isotherm of doxorubicin on magnetite coated with citrate ions (1) and silicon dioxide (2)

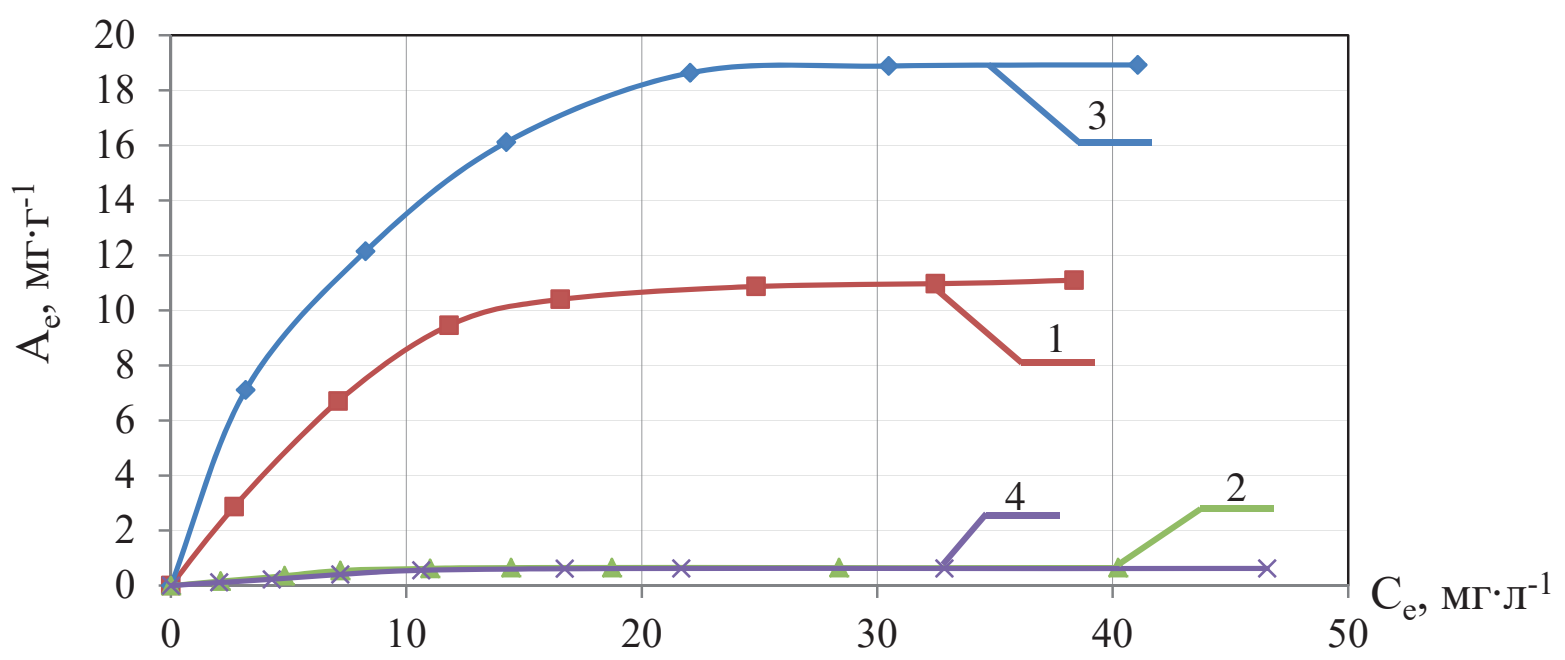

Puс. 7. Изотерлы адсорбции ионов: $\mathrm{Ni}^{2+}$ (1) и $\mathrm{CrO}_{4}{ }^{2-}$ (2) на нанопорошках магнетита, покрытых иитрат-ионами; $\mathrm{Ni}^{2+}$ (3) и $\mathrm{CrO}_{4}{ }^{2-}$ (4) на нанопорошках магнетита, покрытых диоксидом кремния

Fig. 7. Adsorption isotherms of $\mathrm{Ni}^{2+}(1)$ and $\mathrm{CrO}_{4}{ }^{2-}$ (2) ions on magnetite nanopowders coated with citrate ions; $\mathrm{Ni}^{2+}(3)$ and $\mathrm{CrO}_{4}{ }^{2-}(4)$ ions magnetite nanopowders coated with silicon dioxide

для противоопухолевого препарата DOX, также образующего в воде катионы по схеме:

$$
\mathrm{C}_{27} \mathrm{H}_{29} \mathrm{NO}_{11} \cdot \mathrm{HCl} \rightarrow\left[\mathrm{C}_{27} \mathrm{H}_{29} \mathrm{NO}_{11}\right] \mathrm{H}^{+}+\mathrm{Cl}^{-} \text {. }
$$

В соответствии со структурой эозина, диссоциирующего в водном растворе согласно уравнению:

$$
\mathrm{Na}_{2}\left[\mathrm{C}_{20} \cdot \mathrm{H}_{6} \mathrm{O}_{5} \mathrm{Br}_{4}\right] \rightarrow 2 \mathrm{Na}^{+}+\left[\mathrm{C}_{20} \mathrm{H}_{6} \mathrm{O}_{5} \mathrm{Br}_{4}\right]^{2-},
$$

сорбция на синтезированных частицах обоих типов не произошла из-за одинаковых зарядов активных центров поверхности адсорбентов и адсорбатов. Следовательно, механизм процесса адсорбции органических и неорганических ионов на поверхности сорбентов соответствует электростатическому. О том же свидетельствуют косвенные данные, приведенные в работе [28].

Полученные изотермы адсорбции были обработаны в координатах уравнений Ленгмюра и
Фрейндлиха [27], в табл. 2 приведены параметры адсорбции.

Из рассчитанных коэффициентов корреляции следует, что данные изотермы описываются уравнением Ленгмюра, то есть на поверхности магнетита образуется мономолекулярный слой [29]. Определена максимальная сорбционная емкость для всех катионов. Согласно данным табл. 2 для $\mathrm{HЧM} / \mathrm{SiO}_{2}$ она примерно 2 раза выше, чем для НЧМ. Определено число активных центров: $1,6 \cdot 10^{19} ; 2,8 \cdot 10^{19}$ для НЧМ и $\mathrm{HЧM} / \mathrm{SiO}_{2}$, соответственно. Следовательно, модифицирование магнетита диоксидом кремния приводит к повышению сорбционной ёмкости для МГ, DOХ и ионов никеля за счёт увеличения числа активных адсорбционных центров поверхности. 
Таблица 2. Параметры адсорбции ионов на НЧМ и $\mathrm{HЧM}_{\mathrm{S}} \mathrm{SiO}_{2}$

Table 2. $\quad$ Parameters of ion adsorptions on $\mathrm{MNP}$ and $\mathrm{MNP} / \mathrm{SiO}_{2}$

\begin{tabular}{|c|c|c|c|c|}
\hline \multicolumn{3}{|c|}{$\begin{array}{r}\text { Адсорбат } \\
\text { Adsorbate }\end{array}$} & \multirow{2}{*}{$\begin{array}{l}\text { HЧM } \\
\text { MNP } \\
8,24\end{array}$} & \multirow{2}{*}{$\begin{array}{c}\mathrm{HUM} / \mathrm{SiO}_{2} \\
\mathrm{MNP} / \mathrm{SiO}_{2}\end{array}$} \\
\hline \multirow{6}{*}{ 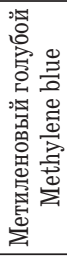 } & \multirow{3}{*}{$\begin{array}{c}\text { Модель } \\
\text { Ленгмюра } \\
\text { Langmuir Model }\end{array}$} & $A_{\max }, \mathrm{M \Gamma} \cdot \Gamma^{-1} / \mathrm{mg} \cdot \mathrm{g}^{-1}$ & & \\
\hline & & $K, \pi \cdot \mathrm{M \Gamma}^{-1} / \mathrm{l} \cdot \mathrm{mg}^{-1}$ & 0,95 & 0,36 \\
\hline & & $R^{2}$ & 0,99 & 0,99 \\
\hline & \multirow{3}{*}{$\begin{array}{c}\text { Модель } \\
\text { Фрейндлиха } \\
\text { Freundlich Model }\end{array}$} & $K_{F}$ & 4,04 & 3,57 \\
\hline & & $n$ & 1,99 & 2,44 \\
\hline & & $R^{2}$ & 0,95 & 0,89 \\
\hline \multirow{6}{*}{ 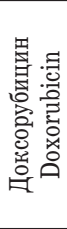 } & \multirow{3}{*}{$\begin{array}{c}\text { Модель } \\
\text { Ленгмюра } \\
\text { Langmuir Model }\end{array}$} & $A_{\max }, \mathrm{M \Gamma} \cdot \Gamma^{-1} / \mathrm{mg} \cdot \mathrm{g}^{-1}$ & 8,56 & 18,76 \\
\hline & & $K, \mathrm{~L} \cdot \mathrm{M \Gamma}^{-1} / \mathrm{l} \cdot \mathrm{mg}^{-1}$ & 0,83 & 0,51 \\
\hline & & $R^{2}$ & 0,99 & 0,99 \\
\hline & \multirow{3}{*}{$\begin{array}{c}\text { Модель } \\
\text { Фрейндлиха } \\
\text { Freundlich Model }\end{array}$} & $K_{F}$ & 3,43 & 6,11 \\
\hline & & $n$ & 1,36 & 2,46 \\
\hline & & $R^{2}$ & 0,97 & 0,94 \\
\hline \multirow{6}{*}{$\mathrm{Ni}^{2+}$} & \multirow{3}{*}{\begin{tabular}{|c|} 
Модель \\
Ленгмюра \\
Langmuir Model
\end{tabular}} & $A_{\max }, \mathrm{M \Gamma} \cdot \Gamma^{-1} / \mathrm{mg} \cdot \mathrm{g}^{-1}$ & 13,59 & 22,32 \\
\hline & & $K, \mathrm{~J} \cdot \mathrm{MI}^{-1} / \mathrm{l} \cdot \mathrm{mg}^{-1}$ & 0,14 & 0,16 \\
\hline & & $R^{2}$ & 0,98 & 0,99 \\
\hline & \multirow{3}{*}{$\begin{array}{c}\text { Модель } \\
\text { Фрейндлиха } \\
\text { Freundlich Model }\end{array}$} & $K_{F}$ & 2,23 & 4,97 \\
\hline & & $n$ & 2,03 & 2,52 \\
\hline & & $R^{2}$ & 0,86 & 0,93 \\
\hline
\end{tabular}

таблица 3. Максилальная сорбционная ёмкость для различных адсорбатов, полученная в данной работе и другими авторали

Table 3. Maximum sorption capacity for various adsorbates obtained in this work and by other authors

\begin{tabular}{|c|c|c|}
\hline 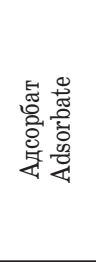 & 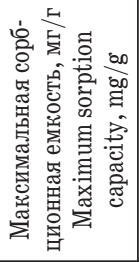 & $\begin{array}{l}\text { Адсорбент } \\
\text { Adsorbent }\end{array}$ \\
\hline \multirow{3}{*}{$\mathrm{Ni}^{2+}$} & 22,32 & $\begin{array}{l}\text { Магнетит покрытый } \mathrm{SiO}_{2} \text { (данная работа) } \\
\text { Magnetite coated with } \mathrm{SiO}_{2} \text { (this work) }\end{array}$ \\
\hline & 1,25 & $\begin{array}{l}\text { Природный магнетит [2] } \\
\text { Mineral magnetite }\end{array}$ \\
\hline & 11,53 & $\begin{array}{l}\text { Магнетит, обработанный соляной кислотой [1] } \\
\text { Magnetite treated with hydrochloric acid }\end{array}$ \\
\hline \multirow{3}{*}{$\mathrm{CrO}_{4}{ }^{2-}$} & 0,75 & $\begin{array}{l}\text { Магнетит покрытый } \mathrm{SiO}_{2} \text { (данная работа) } \\
\text { Magnetite coated with } \mathrm{SiO}_{2} \text { (this work) }\end{array}$ \\
\hline & 0,1 & $\begin{array}{l}\text { Природный магнетит [2] } \\
\text { Mineral magnetite }\end{array}$ \\
\hline & 3,55 & $\begin{array}{l}\text { Магнетит, обработанный соляной кислотой [1] } \\
\text { Magnetite treated with hydrochloric acid }\end{array}$ \\
\hline \multirow{2}{*}{ 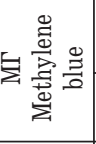 } & 14,73 & $\begin{array}{l}\text { Магнетит покрытый } \mathrm{SiO}_{2} \text { (данная работа) } \\
\text { Magnetite coated with } \mathrm{SiO}_{2} \text { (this work) }\end{array}$ \\
\hline & 43,82 & $\begin{array}{l}\text { Магнетит/графен [15] } \\
\text { Magnetite/graphene }\end{array}$ \\
\hline \multirow{2}{*}{ 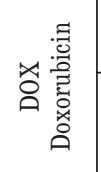 } & 18,76 & $\begin{array}{l}\text { Магнетит покрытый } \mathrm{SiO}_{2} \text { (данная работа) } \\
\text { Magnetite coated with } \mathrm{SiO}_{2} \text { (this work) }\end{array}$ \\
\hline & 53,21 & $\begin{array}{l}\text { Магнетит, модифицированный } \\
\text { поливиниловым спиртом [14] } \\
\text { Magnetite modified with polyvinyl alcohol }\end{array}$ \\
\hline
\end{tabular}

В табл. 3, для сравнения, приведены максимальные адсорбционные емкости для $\mathrm{Ni}^{2+}, \mathrm{CrO}_{4}^{2-}$, $\mathrm{M \Gamma}, \mathrm{DOX}$, полученные на магнетите в данной работе и другими авторами.

Как видно из табл. 3, эти величины имеют разные значения, тем не менее прямое сравнение полученных данных с результатами других авторов затруднено, в связи с тем, что исследования проводятся в неодинаковых условиях и, кроме того, магнетит имеет разные модифицированные поверхности.

\section{Выводы}

1. Получены наночастицы магнетита, состоящие из кристаллического ядра $\mathrm{Fe}_{3} \mathrm{O}_{4}$ с модифицированной поверхностью цитрат-ионами и диоксидом кремния. Полученные частицы имеют узкое распределение по размерам от 10-20 нм. Определена намагниченность насыщения наночастиц магнетита для образцов, покрытых цитрат-ионами, она составляет $92 \mathrm{~A} \cdot \mathrm{M}^{2} /$ кг. После нанесения слоя $\mathrm{SiO}_{2}$, намагниченность насыщения уменьшается до $57 \mathrm{~A} \cdot \mathrm{M}^{2} /$ кг из-за диэлектрического слоя диоксида кремния.

2. Модифицирование поверхности магнетита приводит к изменению знака заряда активных центров поверхности, это подтверждено экспериментально. Установлено, что взаимодействие между поверхностью магнетита и адсорбатом носит электростатический характер. Полученные результаты дают возможность моделировать процессы адсорбции в процессах водоочистки.

3. Изотермы сорбции для ионов $\mathrm{Ni}^{2+}$, метиленового голубого и доксорубицина на модифицированном магнетите аппроксимируются уравнением Ленгмюра, это свидетельствует о том, что адсорбция локализована на отдельных адсорбционных центрах с образованием мономолекулярного слоя.

4. Порошки, модифицированные диоксидом кремния, обладают максимальной сорбционной емкостью по $\mathrm{Ni}^{2+}-22,3 \mathrm{мг} / г$, метиленовому голубому 14,7 мг/г, доксорубицину - 18,8 мг/г. Максимальная сорбционная емкость магнетита, покрытого диоксидом кремния, возросла приблизительно в 2 раза по сравнению с магнетитом, покрытым цитрат ионами.

5. Полученные результаты дают возможность использовать магнетит в качестве сорбента в процессах водоочистки от неорганических, органических соединений и утилизации фармацевтических препаратов на фармацевтических предприятиях и в онкологических центрах

Работа выполнена в ралках Програмлы повышения конкурентоспособности Томского политехнического университета. 


\section{СПИСОК ЛИТЕРАТУРЫ}

1. Sharma Y.C., Srivastava V. Comparative Studies of Removal of $\mathrm{Cr}(\mathrm{VI})$ and $\mathrm{Ni}$ (II) from Aqueous Solutions by Magnetic Nanoparticles // Journal of Chemical and Engineering Data. - 2011. V. 56. - P. 819-825.

2. Мартемьянов Д.В., Галанов А.И., Юрмазова Т.А. Определение сорбционных характеристик различных минералов при извлечении ионов $\mathrm{As}^{5+}, \mathrm{Cr}^{6+}, \mathrm{Ni}^{2+}$ из водных сред // Фундаментальные исследования. - 2013. - № 8. - С. 666-670.

3. Aghdas Heidari, Habibollah Younesi, Zahra Mehraban. Removal of $\mathrm{Ni}(\mathrm{II}), \mathrm{Cd}(\mathrm{II})$, and $\mathrm{Pb}(\mathrm{II})$ from a ternary aqueous solution by amino functionalized mesoporous and nano mesoporous silica // Chemical Engineering Journal. - 2009. - V. 153. - P. 70-79.

4. Decolourization of textile industry wastewater by the photocatalytic degradation process / C. Hachem, F. Bocquillon, 0. Zahraa, M. Bouchy // Dyes and Pigments. - 2001. - V. 49. - № 2. - P. 117-125.

5. Faisal Ibney Hai, Kazuo Yamamoto, Kensuke Fukushi. Hybrid Treatment Systems for Dye Wastewater // Critical Reviews in Environmental Science and Technology. - 2007. - V. 37. - № 4. - P. 315-377.

6. Mompelat S., Le Bot B., Thomas 0. Occurrence and fate of pharmaceutical products and by-products, from resource to drinking water // Environment International. - 2009. - V. 35. - P. 803-814.

7. Jiefeng Zhang, Victor W.C. Chang, Apostolos Giannis, Jing-Yuan Wang. Removal of cytostatic drugs from aquatic environment: A review // Science of the Total Environment. - 2013. V. 445-446. - P. 281-298.

8. Danilenko N.B., Yurmazova T.A., Tran Tuan Hoang, Nguyen Tuan Anh. Pulsed Electric Discharge in Active Metallic Grains for Water Purification Processes // Procedia Chemistry. - 2015. V. 15 . - P. 292-300.

9. Ebrahiem E. Ebrahiema, Mohammednoor N. Al-Maghrabib, Ahmed R. Mobarkia. Removal of organic pollutants from industrial wastewater by applying photo-Fenton oxidation technology // Arabian Journal of Chemistry. - 2017. - V. 10. - P. 1674-1679.

10. Ferrer 0., Gibert 0., Cortina J.L. Reverse osmosis membrane composition, structure and performance modification by bisulphite, iron (III), bromide and chlorite exposure // Water Research. - 2016. - V. 103. - P. 256-263.

11. Parag R. Gogate, Aniruddha B. Pandit. A review of imperative technologies for wastewater treatment II: hybrid methods // Advances in Environmental Research. - 2004. V. 8. - P. 553-597.

12. The role of dipole interaction in hyperthermia heating colloidal clusters of densely-packed superparamagnetic nanoparticles / Rong Fu, Yuying Yan, Clive Roberts, Zeyu Liu and Yiyi Chen // Scientific reports. - 2018 - V. 8. - № 4704. - P. 1-10.

13. Potential use of superparamagnetic iron oxide nanoparticles for in vitro and in vivo bio imaging of human myoblasts / K.R. Wierzbinski, T. Szymanski, N. Rozwadowska, J.D. Rybka, A. Zimna, T. Zalewski, K. Nowicka-Bauer, A. Malcher, M. Nowaczyk, M. Krupinski, M. Fiedorowicz, P. Bogorodzki, P. Grieb, M. Giersig, M.K. Kurpisz // Scientific reports. - 2018. - V. 8. - № 3682. - P. 1-17.

14. Kayal S., Ramanujan R.V. Doxorubicin loaded PVA coated iron oxide nanoparticles for targeted drug delivery // Materials science and Engineering C. - 2010. - V. 30. - P. 484-490.

15. Lunhong Ai, Chunying Zhang, Zhonglan Chen. Removal of methylene blue from aqueous solution by a solvothermal-synthes- ized graphene/magnetite composite // Journal of Hazardous Materials. - 2011. - V. 192. - P. 1515-1524.

16. Thiol modified $\mathrm{Fe}_{3} \mathrm{O}_{4} @ \mathrm{SiO}_{2}$ as a robust, high effective, and recycling magnetic sorbent for mercury removal / S. Zhang, Y. Zhang, J. Liu, Q. Xu, H. Xiao, X. Wang, H. Xu, J. Zhou // Chemical Engineering Journal. - 2013. - V. 226. - P. 30-38.

17. Hoang Vinh Tran, Lam Dai Tran, Thinh Ngoc Nguyen. Preparation of chitosan/magnetite composite beads and their application for removal of $\mathrm{Pb}(\mathrm{II})$ and $\mathrm{Ni}(\mathrm{II})$ from aqueous solution // Materials science and engineering C. -2010 . - V. 30. - P. 304-310.

18. Magnetic nanoparticles: design and characterization, toxicity and biocompatibility, pharmaceutical and biomedical applications / L.H. Reddy, J.L. Arias, J. Nicolas, P. Couvreur // Chemical Reviews. - 2012. - V. 112. - P. 5818-5878.

19. Biocompatible magnetite nanoparticles with varying silica-coating layer for use in biomedicine: physicochemical and magnetic properties, and cellular compatibility / R.K. Singh, T.H. Kim, K.D. Patel, J.C. Knowles, H.W. Kim // Journal of Biomedical Materials Research. - 2012. - Part A.- P. 1734-1742.

20. Казимирова К.О., Штыков С.Н. Синтез и функционализация магнитных наночастиц магнетита хитозаном // Известия Саратовского университета. Новая серия. Серия Химия. Биология. Экология. - 2018. - Т. 18. - Вып. 2. - С. 126-133.

21. Barrera C., Herrera A.P., Rinaldi C. Colloidal dispersions of monodisperse magnetite nanoparticles modified with poly (ethylene glycol) // The Journal of Colloid and Interface Science. - 2009. V. $329 .-$ P. $107-113$.

22. Aqueous ferrofluid of citric acid coated magnetite particles / A. Goodarzi, Y. Sahoo, M.T. Swihart, P.N. Prasad // Materials Research Society Symposium Proceedings. - 2004. - V. 789. - P. 1-6.

23. Ying Wan, Dongyuan Zhao. On the controllable soft-templating approach to mesoporuous silicates // Chemical reviews. - 2007. V. 107. - № 7. - P. 2821-2860.

24. Synthesis and Characterization of Superparamagnetic $\mathrm{Fe}_{3} \mathrm{O}_{4} @ \mathrm{SiO}_{2}$ Core-Shell Composite Nanoparticles / Meizhen Gao, Wen Li, Jingwei Dong, Zhirong Zhang, Bingjun Yang // World Journal of Condensed Matter Physics. - 2011. - № 1. - P. 49-54.

25. Кирсанова К.А., Темникова С.А., Ворончихина Л.И. Применение индикаторного метода при изучении поверхности частиц расширенного графита // Фундаментальные исследования. 2008. - № 10. - C. 60-61.

26. Шарло Г. Методы аналитической химии. Ч. 2. - М.: Изд-во «Химия», 1969.- 774 с.

27. Фролов Ю.Г. Курс коллоидной химии (Поверхностные явления и дисперсные системы). - М.: Изд-во «Химия», 1982. - 400 с.

28. Исследование механизма адсорбции противоопухолевых лекарств на железокарбидных наночастицах / А.И. Галанов, Т.А. Юрмазова, В.А. Митькина, Г.Г. Савельев, Н.А. Яворовский / / Известия Томского Политехнического Университета. 2010. - T. 317. - № 3. - P. 29-33.

29. Кучерова А.Е., Шубин И.Н., Пасько Т.В. Перспективные сорбенты на основе модифицированного наноструктурами цеолита для очистки водных сред от органических примесей // Российские нанотехнологии. - 2018. - Т. 13. - № 5-6. - С. 113-117.

Поступила 22.05.2019 2.

\section{Информация об авторах}

Чан Туан Хоанг, магистрант Инженерной школы ядерных технологий Национального исследовательского Томского политехнического университета.

Юрмазова T.A., кандидат химических наук, доцент отделения естественных наук Школы базовой инженерной подготовки Национального исследовательского Томского политехнического университета.

Baйтулевич $\boldsymbol{E} . \boldsymbol{A}$., кандидат химических наук, доцент отделения естественных наук Школы базовой инженерной подготовки Национального исследовательского Томского политехнического университета. 


\title{
MAGNETITE WITH MODIFIED SURFACE FOR WATER TREATMENT
}

\author{
Hoang Tran Tuan',
}

cungbinh9327@gmail.com

\section{Tatyana A. Yurmazova', yur-tatyana@yandex.ru}

\author{
Elena A. Vaitulevich', \\ rhodamine@tpu.ru \\ ${ }^{1}$ National Research Tomsk Polytechnic University, \\ 30, Lenin Avenue, Tomsk, 634050, Russia.
}

Relevance. Recently, interest in the synthesis of magnetite nanoparticles has been growing, which is due to the possibility of using it as a sorbent for protecting environment, such as, for purifying wastewater from organic compounds, drugs and inorganic heavy metals. The necessity to improve the efficiency of sorption and study mechanism of sorption makes this problem urgent.

The main aim of the research is to determine the sorption possibility of inorganic and organic ions, as well as drugs on magnetite powders with a modified surface obtained by a chemical method for further use in water purification.

Object: magnetic nanopowders based on $\mathrm{FFe}_{3} \mathrm{O}_{4}$ with modified surface with citrate ions and dioxide silica.

Methods: X-ray phase analysis, inductively coupled plasma atomic emission spectrometry, method of thermal desorption of nitrogen, indicator method.

Results. Magnetic powders based on magnetite $\mathrm{Fe}_{3} \mathrm{O}_{4}$ with a surface coated with citrate ions (MNP) and silicon dioxide $\left(\mathrm{MNP} / \mathrm{SiO}_{2}\right)$ were obtained for sorption of organic and inorganic compounds from aqueous media. Their composition and magnetic properties were studied. It is shown that the obtained composition of magnetite coated with dioxide silica corresponds to the crystalline phase of $\mathrm{Fe}_{3} \mathrm{O}_{4}$ and amorphous phase of $\mathrm{SiO}_{2}$. Furthermore, the saturation magnetization $\mathrm{M}_{5}$ and average surface diameter of particles were determined. The sorption isotherms of organic and inorganic compounds on the surface of obtained nanopowders were described by the Langmuir equation, which indicates the formation of a monomolecular layer on the surface of the sorbents. The maximum sorption capacity of $\mathrm{Ni}^{2+}$, methylene blue and doxorubicin on silicon dioxide-coated magnetite powders was determined, it is 22,3, 14,7 and 18,8 mg/g, respectively. The charge of surface active centers of the magnetite was determined by the indicator method. The obtained results make it possible to simulate sorption of organic, inorganic substances and drugs in water purification and utilization of chemical compounds in pharmaceutical plants and cancer centers.

\section{Key words:}

Adsorption, magnetic nanoparticles, surface modification, organic and non-organic ions, charge of surface active centers.

The research was carried out within the Competitiveness Enhancement Program of Tomsk Polytechnic University.

\section{REFERENCES}

1. Sharma Y.C., Srivastava V. Comparative Studies of Removal of $\mathrm{Cr}(\mathrm{VI})$ and $\mathrm{Ni}(\mathrm{II})$ from Aqueous Solutions by Magnetic Nanoparticles. Journal of Chemical and Engineering Data, 2011, vol. 56, pp. 819-825.

2. Martemiyanov D.V., Galanov A.I., Yurmazova T.A. Determination of sorption characteristics of different minerals in extracting compounds of heavy metals from aqueous media. Fundamental research, 2013, no. 8, pp. 666-670. In Rus.

3. Aghdas Heidari, Habibollah Younesi, Zahra Mehraban. Removal of $\mathrm{Ni}(\mathrm{II}), \mathrm{Cd}(\mathrm{II})$, and $\mathrm{Pb}(\mathrm{II})$ from a ternary aqueous solution by amino functionalized mesoporous and nano mesoporous silica. Chemical Engineering Journal, 2009, vol. 153, pp. 70-79.

4. Hachem C., Bocquillon F., Zahraa 0., Bouchy M. Decolourization of textile industry wastewater by the photocatalytic degradation process. Dyes and Pigments, 2001, vol. 49, no. 2, pp. 117-125.

5. Faisal Ibney Hai , Kazuo Yamamoto, Kensuke Fukushi. Hybrid Treatment Systems for Dye Wastewater. Critical Reviews in Envi ronmental Science and Technology, 2007, vol. 37, no. 4, pp. 315-377.

6. Mompelat S., Le Bot B., Thomas 0. Occurrence and fate of pharmaceutical products and by-products, from resource to drinking water. Environment International, 2009, vol. 35, pp. 803-814.

7. Jiefeng Zhang, Victor W.C. Chang, Apostolos Giannis, Jing-Yuan Wang. Removal of cytostatic drugs from aquatic environment: a review. Science of the Total Environment, 2013, vol. 445-446, pp. 281-298.

8. Danilenko N.B., Yurmazova T.A., Tran Tuan Hoang, Nguyen Tuan Anh. Pulsed Electric Discharge in Active Metallic Grains for Water Purification Processes. Procedia Chemistry, 2015, vol. 15, pp. 292-300.

9. Ebrahiem E. Ebrahiema, Mohammednoor N. Al-Maghrabib, Ahmed R. Mobarkia. Removal of organic pollutants from industrial wastewater by applying photo-Fenton oxidation technology. Arabian Journal of Chemistry, 2017, vol. 10, pp. 1674-1679.

10. Ferrer 0., Gibert 0., Cortina J.L. Reverse osmosis membrane composition, structure and performance modification by bisulphite, iron (III), bromide and chlorite exposure. Water Research, 2016, vol. 103, pp. 256-263.

11. Parag R. Gogate, Aniruddha B. Pandit. A review of imperative technologies for wastewater treatment II: hybrid methods. $A d$ vances in Environmental Research, 2004, vol. 8, pp. 553-597.

12. Rong Fu, Yuying Yan, Clive Roberts, Zeyu Liu and Yiyi Chen. The role of dipole interaction in hyperthermia heating colloidal clusters of densely-packed superparamagnetic nanoparticles. Scientific reports, 2018, vol. 8, no. 4704, pp. 1-10.

13. Wierzbinski K.R., Szymanski T., Rozwadowska N., Rybka J.D., Zimna A., Zalewski T., Nowicka-Bauer K., Malcher A., Nowaczyk M., Krupinski M., Fiedorowicz M., Bogorodzki P., Grieb P., Giersig M., Kurpisz M.K. Potential use of superparamagnetic 
iron oxide nanoparticles for in vitro and in vivo bioimaging of human myoblasts. Scientific reports, 2018, vol. 8, no. 3682 , pp. 1-17.

14. Kayal S., Ramanujan R.V. Doxorubicin loaded PVA coated iron oxide nanoparticles for targeted drug delivery. Materials science and Engineering $C$, 2010, vol. 30, pp. 484-490.

15. Lunhong Ai, Chunying Zhang, Zhonglan Chen. Removal of methylene blue from aqueous solution by a solvothermal-synthesized graphene/magnetite composite. Journal of Hazardous Mate rials, 2011, vol. 192, pp. 1515-1524.

16. Zhang S., Zhang Y., Liu J., Xu Q., Xiao H., Wang X., Xu H., Zhou J. Thiol modified $\mathrm{Fe}_{3} \mathrm{O}_{4} @ \mathrm{SiO}_{2}$ as a robust, high effective, and recycling magnetic sorbent for mercury removal. Chemical Engineering Journal, 2013, vol. 226, pp. 30-38.

17. Hoang Vinh Tran, Lam Dai Tran, Thinh Ngoc Nguyen. Preparation of chitosan/magnetite composite beads and their application for removal of $\mathrm{Pb}$ (II) and $\mathrm{Ni}$ (II) from aqueous solution. Materials science and engineering $C, 2010$, vol. 30, pp. 304-310.

18. Reddy L.H., Arias J.L., Nicolas J., Couvreur P. Magnetic nanoparticles: design and characterization, toxicity and biocompatibility, pharmaceutical and biomedical applications. Chemical Reviews, 2012, vol. 112, pp. 5818-5878.

19. Singh R.K., Kim T.H., Patel K.D., Knowles J.C., Kim H.W. Biocompatible magnetite nanoparticles with varying silica-coating layer for use in biomedicine: physicochemical and magnetic properties, and cellular compatibility. Journal of Biomedical Materials Research-Part. A, 2012, vol. 100A, no. 7, pp. 1734-1742.

20. Kazimirova K.0., Shtykov S.N. Synthesis and functionalization of magnetic magnetite nanoparticles with chitosan. News of Saratou University. New series. Chemistry series. Biology. Ecology, 2018, vol. 18, no. 2, pp. 126-133. In Rus.

21. Barrera C., Herrera A.P., Rinaldi C. Colloidal dispersions of monodisperse magnetite nanoparticles modified with poly (ethylene glycol). The Journal of Colloid and Interface Science, 2009, vol. 329, pp. 107-113.

22. Goodarzi A., Sahoo Y., Swihart M.T., Prasad P.N. Aqueous ferrofluid of citric acid coated magnetite particles. Materials Research Society Symposium Proceedings, 2004, vol. 789, pp. 1-6.

23. Ying Wan, Dongyuan Zhao. On the controllable soft-templating approach to mesoporuous silicates. Chemical reviews, 2007, vol. 107, no. 7, pp. 2821-2860.

24. Meizhen Gao, Wen Li, Jingwei Dong, Zhirong Zhang, Bingjun Yang. Synthesis and Characterization of Superparamagnetic $\mathrm{Fe}_{3} \mathrm{O}_{4} @ \mathrm{SiO}_{2}$ Core-Shell Composite Nanoparticles. World Journal of Condensed Matter Physics, 2011, no. 1, pp. 49-54.

25. Kirsanova K.A., Temnikova S.A., Voronchikhina L.I. The use of indicator method in the study of the surface of expanded graphite particles. Fundamental research, 2008, no. 10, pp. 60-61. In Rus.

26. Charlo G. Metody analiticheskoy khimii. Ch. 2 [Methods of analytical chemistry. P. 2]. Moscow, Khimiya Publ., 1969. 774 p.

27. Frolov Yu.G. Kurs kolloidnoy khimii (poverkhnostnye yavleniya idisperstnye sistemy) [Colloid Chemistry Surface Phenomena and Disperse Systems]. Moscow, Khimiya Publ., 1988. 400 p.

28. Galanov A.I., Yurmazova T.A., Mitkina V.A., Saveliev G.G., Yavorovsky N.A. Study of the mechanism of adsorption of anticancer drugs on iron-carbide nanoparticles. Bulletin of the Tomsk $\mathrm{P}_{0}$ lytechnic University, 2010, vol. 317, no. 3, pp. 29-33. In Rus.

29. Kucherova A.E., Shubin I.N., Pasko T.V. Promising sorbents based on modified zeolite with nanostructures for the purification of aqueous media from organic impurities. Russian nanotechnologies, 2018, vol. 13, no. 5-6, pp. 113-117. In Rus.

Received: 22 May 2019.

\section{Information about the authors}

Hoang Tran Tuan, master student, National Research Tomsk Polytechnic University.

Tatyana A. Yurmazova, Cand. Sc., associate professor, National Research Tomsk Polytechnic University.

Elena A. Vaitulevich, Cand. Sc., associate professor, National Research Tomsk Polytechnic University. 